Pramāna, Vol. 16, No. 2, February 1981, pp. 139-146. (c) Printed in India.

\title{
Continuous quantization procedure in quasiclassical scattering: Application to atom-Morse oscillator collisions
}

\author{
RAMAKRISHNA RAMASWAMY* \\ Department of Chemistry, University of North Carolina, Chapel Hill, North Carolina \\ 27514, USA \\ *Present address: Chemical Physics Group, Tata Institute of Fundamental Research, \\ Homi Bhabha Road, Bombay 400 005, India \\ MS received 1 September 1980

\begin{abstract}
The recently developed method of continuous quantization is applied to the atom-Morse oscillator collinear collision problem. This geometrical formulation of the classical scattering process allows for the extraction of transition probabilities for classically forbidden processes with reasonable accuracy. The accuracy of this method over the simple (histogram) quasi-classical procedure is demonstrated
\end{abstract} \\ for two-model systems.
}

Keywords. Quasiclassical scattering; collinear collisions; continuous quantization; atom-Morse oscillator collisions.

\section{Introduction}

Purely classical methods have been widely used in understanding the basic features of inelastic and reactive molecular scattering processes (Porter and Raff 1976). Quantitative accuracy can often be achieved using classical trajectory methods when average or total transition probabilities are desired for problems of chemical interest; in the computation of state-to-state quantities, however, the quasiclassical histogram (QCH) approach (LaBudde and Bernstein 1973) suffers from being statistics-dependent and its inability to treat classically forbidden, but quantum-mechanically allowed processes.

We have recently devised a continuous quantization (CQ) procedure (Ramaswamy and DePristo 1981a) that retains the simplicity of the QCH method and successfully extracts transition probabilities for classically forbidden processes. In this paper, the CQ method is applied to the collinear atom-Morse oscillator problem. The basic feature of this method is the implementation of a continuity ansatz in the standard classical-quantum correspondence principle, and represents an initial attempt at improving the QCH method. (That classical trajectories can be used to obtain quantum information is well-known-this is the classical $S$-matrix (CSM) theory (Marcus 1970; Miller 1970). In all but the simplest cases, the CSM method involves multidimensional root-searches or analytic continuation into the complex plane and is thus intractable for typical chemical problems). One major problem of CQ is the inability to impose proper boundary conditions. However, the wide range of applicability of purely classical methods and the ease in their implementation makes CQ an attractive and useful improvement to the QCH procedure. 
In $\S 2$, the details of the theory and the geometric interpretation of the classical scattering problem is presented, followed by numerical results for two-model collinear scattering problems in $\S 3$. A discussion of the salient features and shortcomings of $\mathrm{CQ}$ is given in $\S 4$.

\section{The method of continuous quantization}

The Hamiltonian for the collinear Morse oscillator-atom scattering system is given, in reduced units (Clark and Dickinson 1973), as

$$
H=H_{0}\left(p_{1}, q_{1}\right)+\frac{p_{2}^{2}}{2 \mu}+V\left(q_{1}, q_{2}\right)
$$

with the internal Hamiltonian of the oscillator as

$$
\begin{aligned}
& H_{0}\left(p_{1}, q_{1}\right)=\frac{1}{2} p_{1}^{2}+D\left[\exp \left(-q_{1} /(2 D)^{1 / 2}\right)-1\right]^{2} \\
& \text { and } V=\exp \left[\alpha\left(q_{1}-q_{2}\right)\right] .
\end{aligned}
$$

The reduced mass of the system is $\mu ; q_{1}$ and $q_{2}$ are the internal and scattering coordinates, $p_{1}$ and $p_{2}$ their conjugate momenta, and $D$, the Morse potential welldepth.

In applying either the QCH or CQ methods, we first transform to the action-angle variables for the Morse oscillator (Rankin and Miller 1971),

$$
\left(p_{1}, q_{1}\right) \rightarrow(I, \theta)
$$

Explicitly, the transformation is

$$
\begin{aligned}
& q_{1}=(2 D)^{1 / 2} \ln \left\{\lambda^{-2}\left[1-\left(1-\lambda^{2}\right)^{1 / 2} \cos \theta\right]\right\}, \\
& p_{1}=\frac{(2 D)^{1 / 2} \lambda\left(1-\lambda^{2}\right)^{1 / 2} \sin \theta}{1-\left(1-\lambda^{2}\right)^{1 / 2} \cos \theta}
\end{aligned}
$$

where $\lambda=1-I /(2 D)$.

Thus $\quad H_{0}\left(p_{1}, q_{1}\right) \rightarrow I-I^{2} /(4 D)=H_{0}(I)$,

and $\quad H \rightarrow H_{0}(I)+p_{2}^{2} / 2 \mu+V\left(I, \theta, q_{2}\right)$.

Asymptotically, $q_{2}$ is chosen such that $V\left(I, \theta, q_{2}\right)$ is negligibly small and the initial oscillator action $I_{i}$ is chosen to be a half integer*, to correspond to an initial stationary quantum state by the usual Böhr-Sommerfeld quantization condition (Miller 1974).

*The definition of the action variable here differs from the usual definition by a factor $\frac{1}{2} \pi$ and integer". The more proper phrase $(\hbar=1)$, it is convenient to use terminology such as " half defined actions) here and elsewhere in the text. "half-integral multiple of $n$ " (with the presently 
The initial value of $p_{2}$ is determined from the total energy. A uniform distribution of initial angles, $\theta_{i}$ in $(0,2 \pi)$ gives different trajectories which are integrated according to Hamiltons equations for (5)

$$
\begin{array}{ll}
\dot{I}=-(\partial V / \partial \theta), & \dot{p}_{2}=-\left(\partial V / \partial q_{2}\right), \\
\dot{\theta}=\frac{\partial H_{0}}{\partial I}+\frac{\partial V}{\partial I}, & \dot{q}_{2}=p_{2} / \mu .
\end{array}
$$

Asymptotically, when $\dot{I} \sim 0$, the final action $I_{f}$ which is usually not a half-integer needs to be quantized according to some prescription. In the $\mathrm{QCH}$, this amounts to assigning it to a bin in action space, of width $\hbar$, centred about the half-integer actions. In the $\mathrm{CQ}$ method, however, correspondence is made between this final arbitrary (i.e. not necessarily a half-integer) action, and a non-stationary quantum state.

It is useful to consider a mapping $\phi$ that associates the action $I=(n+1 / 2) \hbar$ with a quantum state $\phi_{n}$. This is particularly simple in action-angle quantum mechanics (Augustin and Rabitz 1979),

$$
(I, \theta) \rightarrow\left(\frac{1}{2 \pi}\right)^{1 / 2} \exp \left(-i I_{n} \theta_{n} / \hbar\right)
$$

The correspondence is in essence between the classical flow of the Hamiltonian, $H_{0}$, which for a 1-degree of freedom case is simply a 1-torus (a closed curve) in the (isolated) phase-space of $H_{0}$ and quantum wavefunctions (Percival 1977). Thus, we associate the final action $I_{f}\left(\theta_{i}\right)$, which is a function of the initial angle $\theta_{i}$ with a non-stationary wavefunction by the same mapping $\phi$

$$
\left(I_{f}\left(\theta_{i}\right), \theta_{f}\right) \rightarrow\left(\frac{1}{2 \pi}\right)^{1 / 2} \exp \left(-i I_{f} \theta_{f} / \hbar\right) \equiv \phi^{(f)}
$$

and project onto the stationary states $\phi_{n}$,

$$
\phi^{(f)}=\sum_{n=0}^{\text {open states }} a_{n}\left(\theta_{i}\right) \phi_{n}
$$

Finally, transition probabilities $P_{i \rightarrow n}$ are obtained by

$$
P_{i \rightarrow n}=\frac{1}{2 \pi} \int_{0}^{2 \pi}\left|a_{n}\left(\theta_{i}\right)\right|^{2} d \theta_{i}=\frac{1}{2 \pi} \int_{0}^{2 \pi} d \theta_{i}\left|\left\langle\phi_{n}^{*} \mid \phi^{(f)}\right\rangle\right|^{2} .
$$

Rather than associate the continuous action interval $(n, n+1)$ with its mid-point, and thus with $\phi_{n}$, as is done in QCH, we incorporate higher order terms as wetl with CQ. Since the effect of translational states has been distegarded, however, except 
for the restriction of the summation in (9), to open states, the proper boundary conditions are not met*.

By the QCH procedure, the transition probability is simply given as

$$
P_{\rightarrow n}=N_{n}(i) / N_{T}(i)
$$

where $N_{T}(i)$ is the total number of trajectories with initial action $I_{i}=(i+1 / 2) \hbar$ and $N_{n}(i)$ the number of trajectories with final action in the $n$ bin $\left(i . e . n \hbar \leqslant I_{f} \leqslant\right.$
$(n+1) \hbar)$.

A pictorial representation of the two procedures is shown in figure 1 . The final action $I_{f}$ as a function of the initial angle $\theta_{i}$ is graphed in the central diagram. The points marked $j^{\prime}, k k^{\prime}, m^{\prime}$ correspond to stationary trajectories i.e., those with proper boundary conditions. The CSM theory constructs the scattering matrix from just these trajectories. The upper box shows the effect of the QCH method which assigns the final actions to the respective 'bins'. The CQ method, on the other hand, yields the lowest curve, where the quantity displayed is

$$
I_{f}^{C Q}\left(\theta_{i}\right)=\sum_{n=0}^{\text {open states }}\left|a_{n}\left(\theta_{i}\right)\right|^{2} I_{n} \text {. }
$$

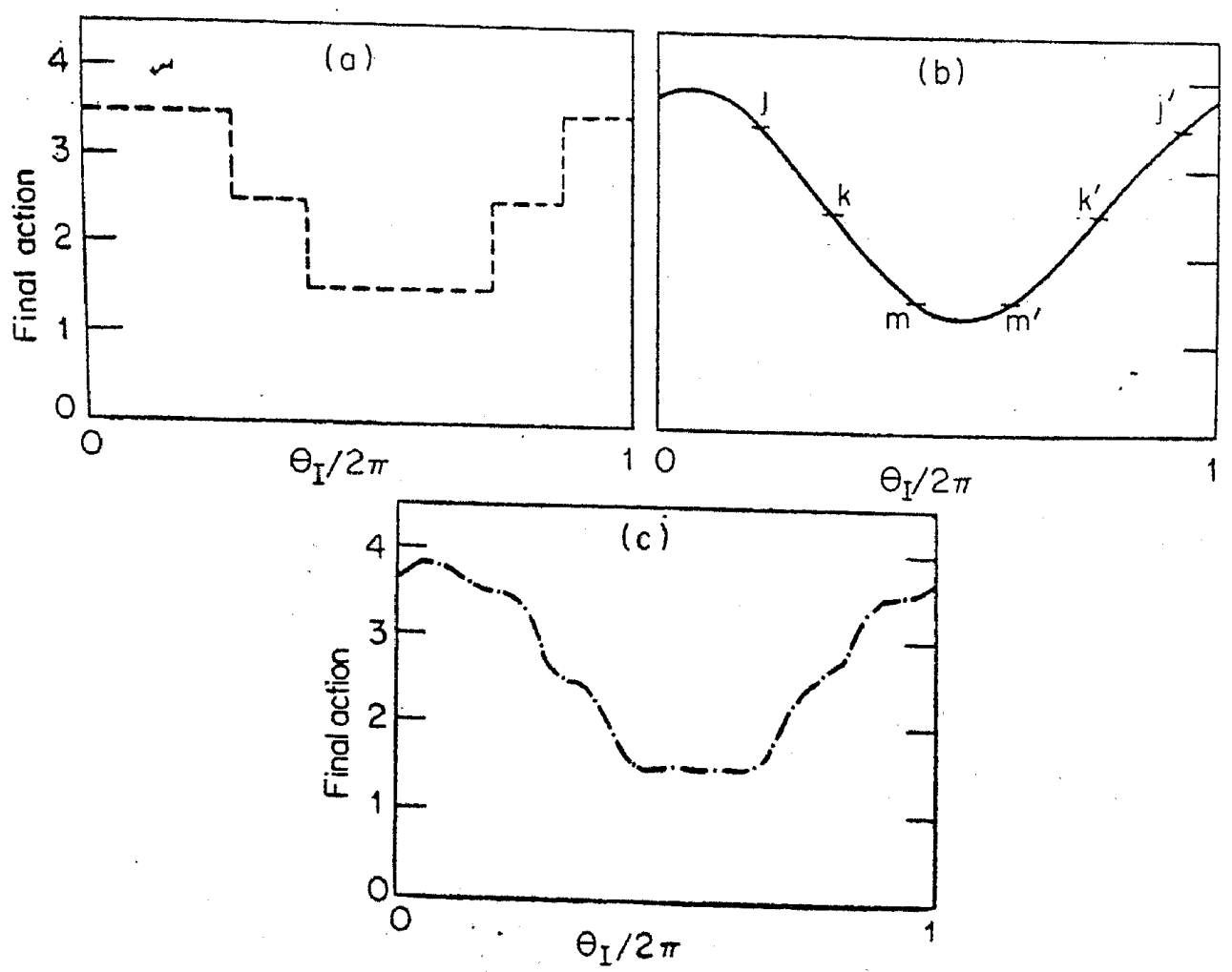

Figure 1. The effective final action as a function of the initial angle variable: (a) histogram method; (b) no quantization; (c) continuous quantization.

*Other problems arising from the restriction $n \geqslant 0$ are discussed in Ramaswamy and
Pristo, 1981a. 
which may be considered as an average final action. It is clear that while the CQ method does not completely decompose the final action curve into proper contributions, due to the neglect of proper boundary conditions, its effect is not as drastic as the $\mathrm{QCH}$ in approximating the true final action.

\section{Application}

Two systems of atom-collinear Morse oscillators are treated here. Accurate quantum mechanical transition probabilities for several model systems are available, (Clark and Dickinson 1977), and it is against these that the results of the CQ and $\mathrm{QCH}$ methods are compared.

For the choice of parameters, $a=0.314 \quad D=9 \cdot 3$ and $\mu=2 / 3$, which corresponds to the $\mathrm{He}+\mathrm{H}_{2}$ collision system, a typical collision mapping is shown in figure 2 . Each trajectory is run for exactly the same duration, so that the transformation of the initial manifold to the final one (which is still a 1-torus, although distorted) due to the collision is evident. This final 1-torus displays the characteristic whorl structure associated with the evolution of closed curves that are not invariant curves of $H_{0}$ (For a discussion of the formation and evolution of whorls in algebraic mappings, see Berry et al 1979). The procedure for obtaining transition probabilities as given in (7) to (10) is direct. The integral in (10) can be evaluated with either WKB type wavefunctions, or approximately, (Ramaswamy and DePristo, 1981a), as

$$
a_{n}\left(\theta_{i}\right)=\frac{1}{2 \pi} \int_{0}^{2 \pi} d \theta \exp \left(-i\left(I_{f}-I_{n}\right) \theta / \hbar\right)
$$

Shown in figure 3 are the transition probabilities $\dot{P}_{n \rightarrow n+\Delta}, n=0,1, \ldots, 5$ and $\Delta= \pm 1, \ldots, \pm 4$ at a total energy of $E=8 \hbar \omega(\hbar=\omega=1$ in these calculations) computed by $\mathrm{CQ}, \mathrm{QCH}$ and close-coupled quantum calculations. For classicallyallowed transitions, the $\mathrm{CQ}$ results are typically better than the $\mathrm{QCH}$ results. For classically forbidden processes, while the $\mathrm{QCH}$ gives zero-transition probabilities, $C Q$ yields results within a factor of 2-3 for probabilities $>10^{-3}$.

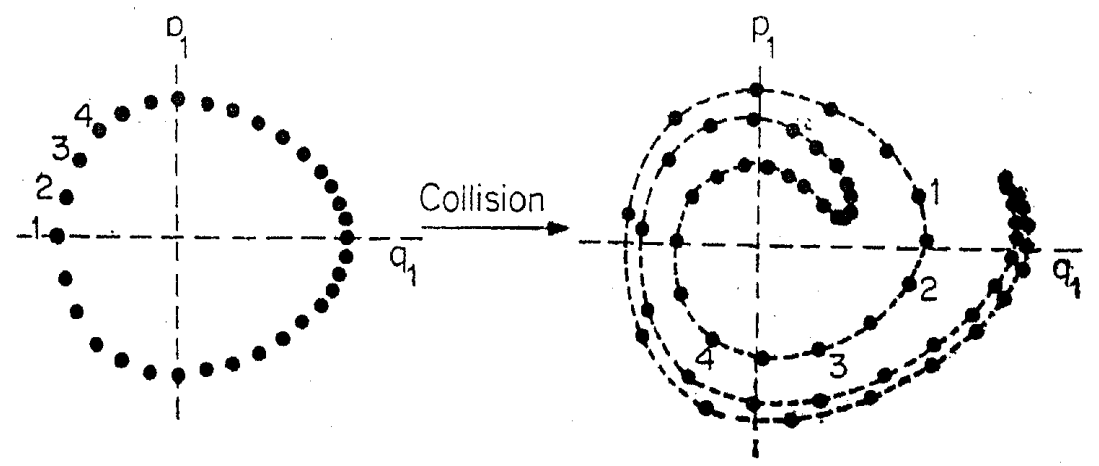

Figure 2. Curve map of the collision process for an atom-Morse oscillator system. The initial phase space curve corresponds to a pure (stationary) quantum state, while the final curve corresponds to a non-stationary state. Some of the initial conditions (and the respective final conditions) are labelled for clarity; 


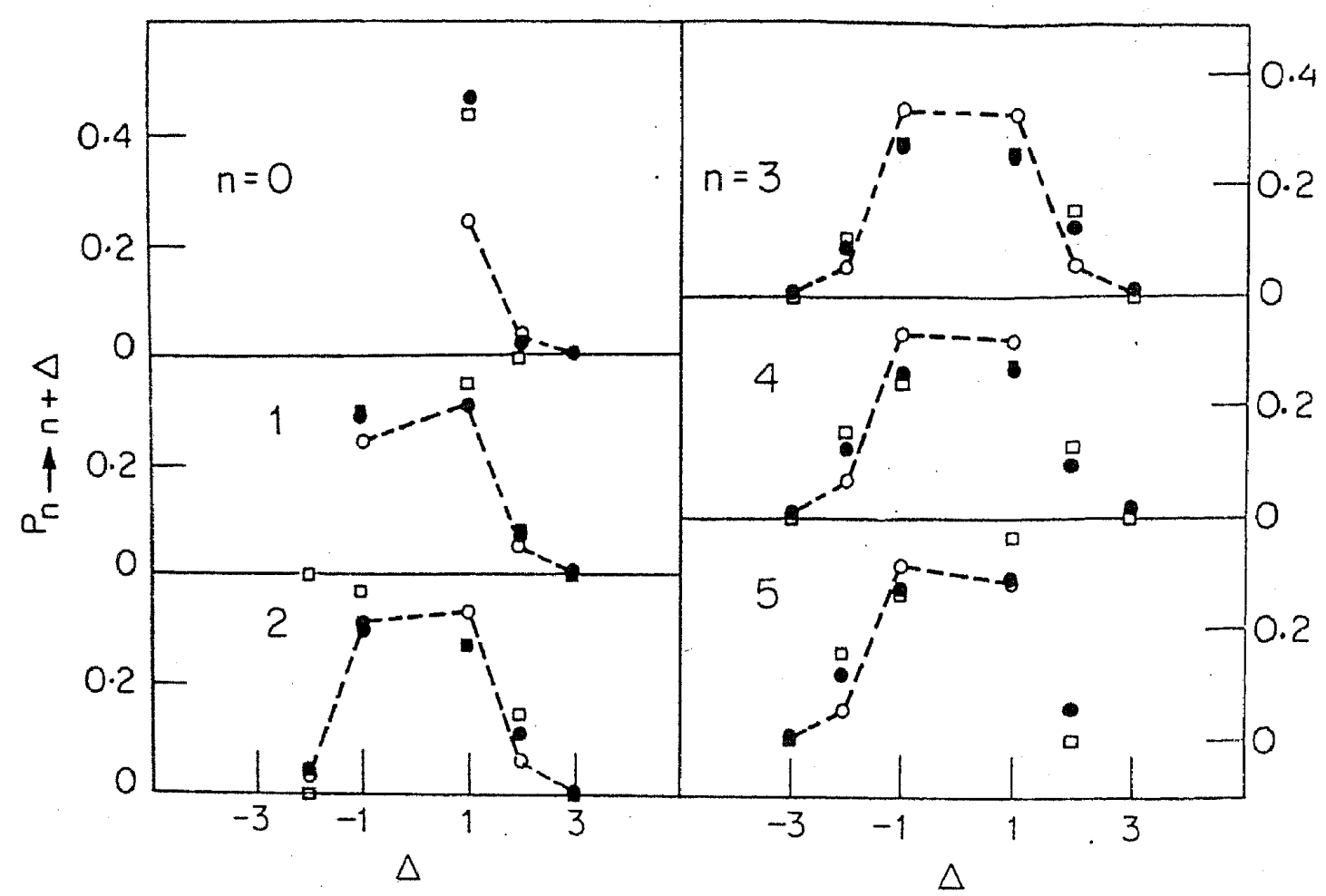

Figure 3. Transition probabilities $P_{n \rightarrow n+\Delta}$ as a function of $n$ and $\Delta$ at a total energy of 8 vibrational quanta for the $\mathrm{He}+\mathrm{H}_{2}$ collision system. Open circles (O) connected by dashed lines are the exact quantum results; open squares ( $\square$ ) are the quasiclassical histogram results, and the filled circles are the present continuous quantization results. The elastic values $\Delta=0$ are not displayed.

The second system studied corresponds to the $\mathrm{Br}_{2}+\left(\mathrm{H}_{2}\right)$ collision system, $\alpha=0.1278, D=75.525$ and $\mu=0.006268$. The quantum results for this system are more in accord with what might be expected in real systems. (In the $\mathrm{He}+\mathrm{H}_{2}$ system, considerable quantum interference, leads to anomalously large propensities for multiquanta transitions.) The present $C Q$ method gives excellent agreement for most of the transitions; $\mathrm{QCH}$ gives nonzero probabilities only for $\Delta= \pm 1$. These results are compared at $E=10 \hbar \omega(\hbar=\omega=1)$ in figure 4 .

\section{Discussion}

The application of the continuous quantization method to the case of atom-Morse oscillator collisions presented here is seen to yield a significant improvement of the standard quasiclassical histogram technique in molecular scattering. The two methods are, however, linked in that $\mathrm{CQ}$ results are excellent when the $\mathrm{QCH}$ results are semiquantitative, but the $\mathrm{CQ}$ results are only marginally an improvement of $\mathrm{QCH}$, when the latter is in very large error for even classically allowed processes. Thus, the present method becomes increasingly accurate with increasing total energy, and with Truhlar 1975). Further, ther forward classical trajectories (see e.g. Duff and is perturbative, either method quantum interference effects are large or the problem 


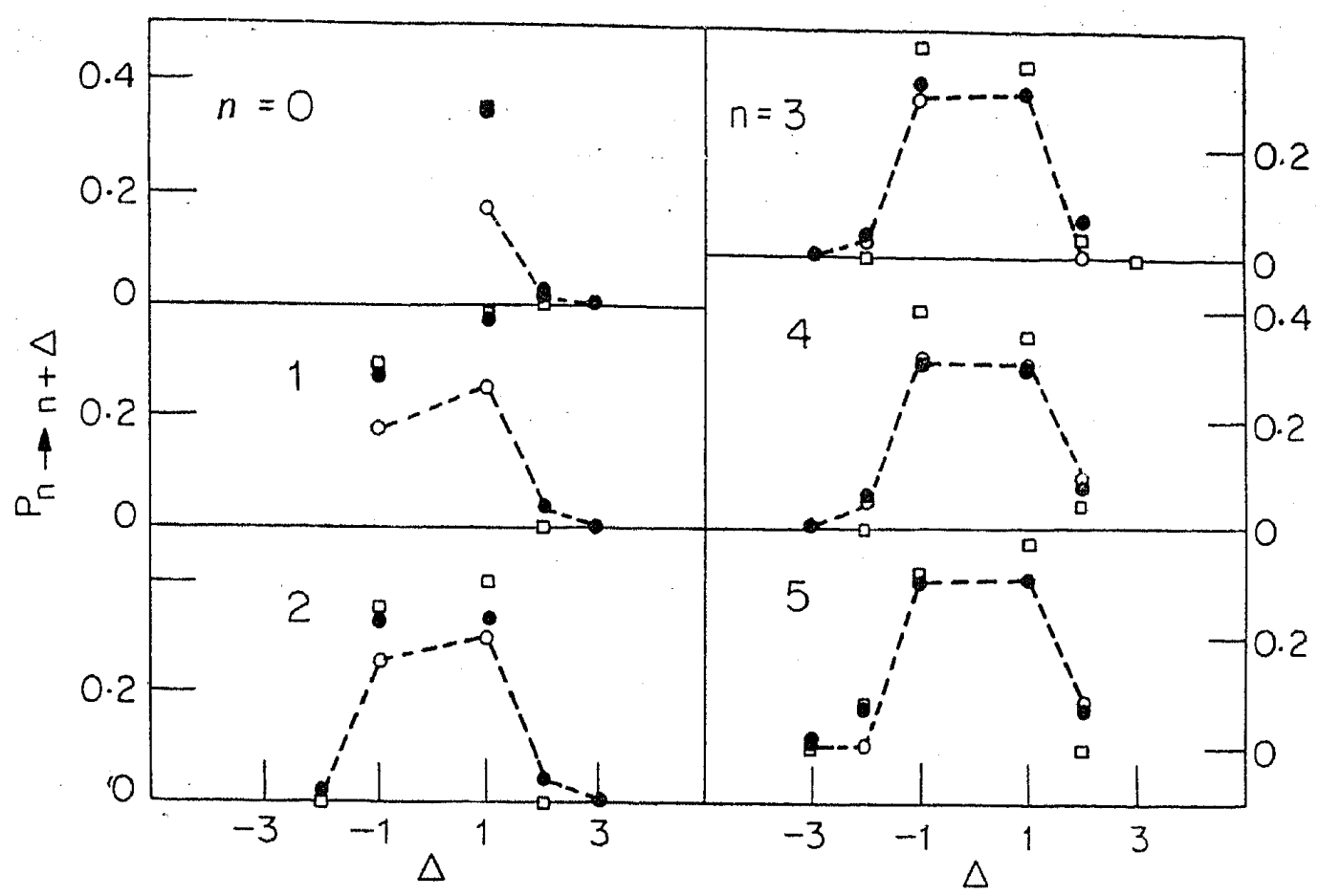

Figure 4. Same as figure 3 , for the $\mathrm{Br}_{2}+\left(\mathrm{H}_{2}\right)$ system at a total energy of 10 vibrational quanta.

The most serious shortcoming, is, however, the lack of proper boundary conditions, which leads to over-estimation of transition probabilities to states that are almost closed. Clearly, further work is necessary to properly account for the translational states, perhaps by a simple box quantization.

In spite of these shortcomings, $\mathrm{CQ}$ greatly increases the information that can be retrieved from a classical trajectory calculation. Application to the collinear atomMorse reactive collision system and a discussion of extensions to three-dimensional and many-degree of freedom systems will be presented elsewhere (Ramaswamy and DePristo 1981b).

\section{Acknowledgement}

This work was supported by a grant from the Research Corporation.

\section{References}

Augustin S and Rabitz H 1979 J. Chem. Phys. 714956

Berry M V, Balazs N L, Tabor M and Voros A 1979 Ann. Phys. 12226

Clark A P and Dickinson A S $1973 J$. Phys. B6 164

Duff J W and Truhlar D G 1975 Chem. Phys. 9243

LaBudde R A and Bernstein R B 1973 J. Chem. Phys. 593687

Marcus R A 1970 Chem. Phys. Lett. 7525

Miller W H 1970 J. Chem. Phys. 531949

Miller W H 1974 Adv. Chem. Phys. 2569 
Percival I 1977 Adv. Chem. Phys. 361

Porter R N and Raff L M 1976 in Dynamics of molecular collisions ed. W H Miller (New York:
Plenum)

Ramaswamy R and DePristo A E 1981a Chem. Phys. Lett. 77190

Ramaswamy $\mathbf{R}$ and DePristo A E $1981 \mathrm{~b}$ to be published

Rankin C C and Miller W H 1971 J. Chem. Phys. 553150 